
\&s
Research Square
Preprints are preliminary reports that have not undergone peer review.
They should not be considered conclusive, used to inform clinical practice, or referenced by the media as validated information.

\title{
Complete Genome sequence of a new variant of jujube mosaic-associated virus isolated from jujube (Ziziphus jujuba Mill.) grown at Aksu in Xinjiang of China
}

Jianyu Bai ( $\sim$ bjyhao2010@sina.com )

Chinese Academy of Forestry Xinjiang Branch https://orcid.org/0000-0002-9172-0702

Baojun Liu

Xinjiang Agricultural University

Guoxin Zhang

Xinjiang Agricultural University

Aixing Gu

Xinjiang Agricultural University

Danbo Song

Xinjiang Agricultural University

Quan Wang

Xinjiang Agricultural University

Hong Li

Chinese Academy of Forestry Xinjiang Branch

\section{Research Article}

Keywords: jujube, genome sequence, badnavirus, mosaic

Posted Date: April 26th, 2021

DOI: https://doi.org/10.21203/rs.3.rs-442593/v1

License: (9) (i) This work is licensed under a Creative Commons Attribution 4.0 International License. Read Full License 


\section{Abstract}

This work reports the discovery and the complete genome sequence of a novel member of the genus Badnavirus in the family Caulimoviridae from a Chinese jujube tree of known variety which grown in Aksu, Xinjiang, China. The symptoms of jujube leaves infected by the virus showed mosaic and malformation, and round chlorotic spots on infected fruits. The genome of this virus is a circular doublestranded DNA, which has the length of $7086 \mathrm{nt}$ and has a genome structure similar to the one reported for jujube mosaic-associated virus (JuMaV), with five open reading frames (ORFs). High nucleotide and amino acid sequence similarity were seen between JuMaV and the new virus, and the nucleotide (NT) difference of the genome was greater than $20 \%$ in the RT/ Rnase H coding region of ORF3. Consequently, the virus was identified a new variant of JuMaV, and propose the name as jujube mosaic-associated virus A (JuMaVA).

\section{Main Text}

The genus Badnavirus is the largest of the eight known Genera of the Caulimoviridae, with nearly twice as many species as all other genera[1]. The genome consists of one circular double-stranded DNA of 6.9 to $9.2 \mathrm{~kb}[2]$, which typically encodes three open reading frames (ORFs), all of which are located on the on the plus strand[3,4]. Badnavirus ORF1 encodes a small protein of unknown function. ORF2 encodes a protein of possesses a conserved coiled-coil motif as virion-associated protein (VAP)[5]. ORF 3 encodes a large polyprotein which is processed into the movement protein (MP), coat protein (CP), aspartic protease (AP), reverse transcriptase (RT) and ribonuclease $H(R N a s e ~ H)[6]$. Moreover, some badnaviruses have more ORFs of unknown function have been reported from other species[7,8]. The RT/ RnaseH coding region of ORF3 is the most conserved region in the genome, and the nucleotide (NT) difference in this part of the genome is greater than $20 \%$, which is used to distinguish the species of this genus[9]. Badnaviruses are spread by vegetative propagation, mealybug vectors, and in some cases by seed[10].

Chinese jujube (Ziziphus jujuba Mill.) is one of the oldest cultivated fruit trees in the world. Its use and cultivation history can be traced back to the Neolithic Age 7000 years ago[11,12]. By 2012, the area of jujube in Xinjiang of china was $345,000 \mathrm{hm}^{2}$, of which Aksu had the largest planting area at 136,000 $\mathrm{hm}^{2}[13]$. In recent years, Jujube mosaic disease (JuMD) is caused by virus infection had been reported many times in China. However, pathogenic mechanism of JuMD needs further research and exploration. Two complete sequences of JUMD can be obtained from the database, jujube mosaic-associated virus (JuMaV, KX852476.1) and jujube associated Badnavirus (JuAB, MN274946.1), respectively. Although sequence analysis of JuMaV has been reported, diseased leaves were collected from Chinese jujube trees grown in Beijing, China[14], there is no information on JuMD in other regions and jujube variety.

In July 2020, leaf and fruit samples were collected from other single plant of Z. jujuba cv. 'Huizao' (sample IDs: HZ, AKS-6) growing areas in Aksu, Xinjiang Uygur Autonomous Region, China. These plants showed symptoms of mosaic and malformation on young leaves (Fig. 1A, left panel), and round chlorotic spots on infected fruits (Fig. 1A, right panel). All leaf and fruit samples were mixed into one pool, 
respectively, from which total RNA was extracted using a TransZol Up Plus RNA Kit (Transgen) for Small RNA and RNA Sequencing analysis.

A cDNA library of sRNAs library was constructed and sequenced on an Illumina HiSeq XTen sequencing machine (Illumina) with a paired-end $150 \mathrm{bp}$ setup[14]. A total of 179 contigs length sequence between 34 to $448 \mathrm{nt}$ were found in leaf and fruit samples, which had high sequence identity ranging from $46.3 \%$ to $94.44 \%$ with several badnaviruses. Therefore, we concluded that the virus was a badna-like virus found in mosaic-diseased jujube trees.

Total DNA was extracted from obviously symptomatic leaves and fruits using DNAsecure Plant Kit. In order to identity the virus, two degenerate primers pair BADNA-FP/RP were used to screen sequence of badnavirus from the total DNA[15], designed to amplify 529 bp sequences in the reverse transcriptase/RNase $\mathrm{H}$ region has been used for taxonomic purposes within this genus[1]. The PCR of leaves and fruits samples both yielded an amplicon of approximately $500 \mathrm{bp}$ were analysed by gel electrophoresis in $1.5 \%(\mathrm{w} / \mathrm{v})$ agarose gels. The amplicons were cloned into the pEASY ${ }^{\circledR}-\mathrm{T} 1$ Cloning vector, and sequenced. BLAST analysis of the sequencing results revealed that the 528-bp PCR fragment shared maximum sequence identity of $81.8 \%$ with the corresponding regions of Jujube mosaicassociated virus (JuMaV, KX852476.1) belong to the genus badnaviruses. Based on the JuMaV alignment, 5 primers were designed spanning the whole genome and used to obtain the full-length genome sequence of this badnavirus (Table 1). PCR amplifications were performed in 25- $\mu$ reaction mixtures containing $1 \mu \mathrm{l}$ of DNA template, $13 \mu \mathrm{l} 2 \times$ Es Taq MasterMix by the manufacturer (CWBIO), $1 \mathrm{mM}$ each primer, $9 \mu \mathrm{l}$ of $\mathrm{ddH}_{2} \mathrm{O}$. PCR cycling was as follows: initial denaturation at $94^{\circ} \mathrm{C}$ for $5 \mathrm{~min}$, followed by 30 cycles of $94^{\circ} \mathrm{C}$ for $30 \mathrm{~s}, 55^{\circ} \mathrm{C}$ for $30 \mathrm{~s}$, and $72^{\circ} \mathrm{C}$ for $1 \mathrm{~min}$, with a final extension at $72^{\circ} \mathrm{C}$ for $10 \mathrm{~min}$. Amplicons were separated by electrophoresis in $1.4 \%$ agarose gels purified by EasyPure ${ }^{\circledR}$ Quick Gel Extraction Kit, cloned into the pEASY ${ }^{\circledR}-\mathrm{T} 1$ Cloning vector and transformation of E. coli DH5a, positive clones were obtained and sequenced by Sangon Biotech (Shanghai) Co., Ltd. In all cases, at least three independent clones in both directions were sequenced to determine the exact sequence of the presumed full-length banavirus genome.

Table 1. Primers used for the amplification of full-length DNAs of JuMaVA from sample HZ and PCR detection for JuMaVA in jujube samples. 


\begin{tabular}{|c|c|c|c|c|}
\hline Primer & Sequence $\left(5^{\prime}-3^{\prime}\right)$ & Position (nt) & Product Size (bp) & Sequence Identities $^{\circ}$ \\
\hline $\mathrm{F} 1_{552-571}$ & agccgtaaagctcacggaag & $552-571$ & 2653 & $99.29 \%$ \\
\hline $\mathrm{R} 1_{2985-3004}$ & ggcagttgccattgttcacc & 2985-3004 & & \\
\hline$F 2_{2959-2978}$ & gagcccgttttcaaggagga & $2959-2978$ & 2017 & $100 \%$ \\
\hline R2 $4914-4933$ & catggtcctgctgacatggt & $4914-4933$ & & \\
\hline $\mathrm{F}_{3542-3561}$ & cccacgaatacacttcgcct & $3542-3561$ & 1585 & $99.66 \%$ \\
\hline $\mathrm{R}_{5028-5047}$ & agcctgtccaaagttcctcg & $5028-5047$ & & \\
\hline$F 4_{5027-5046}$ & tcgaggaactttggacaggc & $5027-5046$ & 2099 & $83.64 \%$ \\
\hline $\mathrm{R} 4_{7013-7032}$ & gcttacacgcaaagcaaacg & 7013-7032 & & \\
\hline$F 5_{6467-6486}$ & tacagaatggcagacgctgg & $6467-6486$ & 1663 & $99.59 \%$ \\
\hline $\mathrm{R}_{715-734}$ & aggtcttgcgaagccaactg & $715-734$ & & \\
\hline
\end{tabular}

a: The sequenced results of primer pairs share highest nucleotide sequence identity with JuMaV (accession KX852476.1)

The edited sequences were used for similarity BLAST searches in the NCBI GenBank databases (http://www.ncbi.nlm.nih.gov/genbank/). Multiple Nucleotide sequences obtained by sequencing were assembled and analyzed using Mega Version7.0. Genome maps were generated using SnapGene® Viewer version4.3.6. Full-length genome sequences were assembled in MEGA version7 and ORFs were predicted using NCBI ORFfinder with minimal ORF length of $75 \mathrm{nt}$ (https://www.ncbi.nlm.nih.gov/orffinder/). Use the NCBI conserved domain tool to search for conserved domains of putative gene products (http://www.ncbi.nlm.nih.gov/Structure/cdd/wrpsb.c

gi).

The five nucleotide sequences obtained through sequenced were analyzed using similarity BLAST searches in the NCBI GenBank database for sequence identities with other badnaviruses(Table1). After assembly, a circular DNA of 7086 bp was obtained, shared nucleotide sequence identity $99.54 \%$ and amino acid sequences identity $94.61 \%$ with JuMaV (accession KX852476.1). This virus was possible a new variant of JuMaV, referred to as jujube mosaic-associated virus A (JuMaVA). The full-length sequence of the circular DNA virus has been registered in GenBank with the accession number MW892537.

Genome and conserved domains analysis, genome structures of JuMaVA were representative of typical members of the genus Badnaviruses (Fig. 1B). Several highly conserved motifs were found in the 
JuMaVA genomes, which had been reported in well-characterized badnavirus genomes[16,17].The JuMaVA genomes contain a tRNA ${ }^{\text {Met }}$ binding site (TGGTATCAGAGC ${ }_{1-12}$ ) which begins at the $5^{\prime}$ end of the viral plus-strand, and occurs in the plant host and serves to initiate viral RNA transcription[18]. There was a TATA box (TATAAATA ${ }_{7002-7009}$ ) and a poly(A) (AATAAAAA 7057-7064 ) signal in the non-coding intergenic region of upstream of the tRNA ${ }^{\text {Met }}$ site. The genome organization contains five ORFs typical for badnaviruses. ORF1, ORF2, ORF4 shared the highest nt $(99.77 \%, 99.51 \%, 100 \%)$ and amino acid $(100 \%$, $99.25 \%, 100 \%$ ) sequence identity with the JuMaV. Same as that of JuMaV, ORF3 was split into ORF3a, ORF3b by a 70-nt intergenic non-coding region, which is highly variable between the viruses[19]. An uncharacterized superfamily domain approximately 200 residues long in ORF1, referred to as 'domain of unknown function (DUF1319)'[20], be restricted to badnaviruses. ORF2 had no conserved domains were identified. The ORF3b of JuMaVA contained four domains: a zinc-finger domain, a pepsin-like aspartate protease domain, an RT-LTR domain, and an RNase H domain. ORF3a contained the 5'-largest domain was the coding region of JuMaVA movement protein (MP).

Nucleotide and amino acid sequences of the the putative JuMaVA RT and RNase $\mathrm{H}$ region were analyzed for sequence identities with other badnaviruses. JuMaVA had the highest sequence identity $(73.93 \%)$ in nucleotide and $84.41 \%$ in amino acid sequences with JuMaV in RT- RNase $\mathrm{H}$ region. Also, the RT-RNase $\mathrm{H}$ and complete genome sequences shared in the range of $69.62-73.93 \%$ and $66.16-74.30 \%$ nt sequence identity with well-characterized badnaviruses, $73.26-80.11 \%$ and $42.30-72.18 \%$ in amino acids.

Phylogenetic trees were generated, which based on full nucleotide sequences of JuMaVA and nucleotide sequences of RT-RNase $\mathrm{H}$ region with neighbour-joining $(\mathrm{NJ})$ method using Mega Version7.0 to determine the taxonomic position of JuMaVA. RT/ RNase H sequences were selected for analysis because they play a critical role in viral replication, therefore are subject to more stringent variability constraints. The fullgenome phylogenetic tree shows that JuMaV is in a group with JuMaV (Fig. 1C). The phylogenic tree based on the RT/RNase H nucleotide sequences indicated that JuMaVA clusters with JuMaV (Fig. 1D). The presence of these hallmark features further support the identification of JuMaVA as badnaviruses. Moreover, JuMaVA shares nucleotide sequence identity $99.5 \%$ with JuMaV in full sequence and $74.25 \%$ in RT- RNase $\mathrm{H}$ region. Based on the difference of the genome is greater than $20 \%$ in RT- RNase $\mathrm{H}$ region, we propose that JuMaVA as a new variant of JuMaV is more accurate.

\section{Conclusion}

In conclusion, the first complete genome sequence of jujube mosaic virus was identified isolated from Chinese Jujube trees in Aksu, Xinjiang, China. Not only did we isolate the virus from diseased leaves, but we also isolated the virus from the symptomatic jujube fruit for the first time. Based on the analysis of full-length genome sequences, the virus was identifed as the closest relative of JuMaV belonging to a member of the badnavirus species in the family Caulimoviridae. We propose this new member of the genus Badnavirus to be designated "Jujube mosaic-associated virus A" (JuMaVA), which is a new variant of JuMaV infecting jujube (Ziziphus jujuba Mill.) grown at Aksu in Xinjiang of China. The work confirmed that the transmission of the virus is widespread and variable. Therefore, we should pay more attention to 
the harm of this virus to jujube trees in daily management. The pathogenic principle and pathogenicity of JuMaVA are the main direction of our research in the future.

\section{Declarations}

Funding This work was supported by the Natural Science Foundation of Xinjiang Uygur Autonomous Region (grant number 2017D01A28); “Excellent Postdoctoral Professionals and General Funding Staff of Living Subsidies" of the Department of Human Resources and Social Security of Xinjiang Uygur Autonomous Region.

\section{Compliance with ethical standards}

Conflict of interest The authors declare that they have no conflict of interest.

Ethical approval This article does not contain any studies with human participants or animals performed by any of the authors.

\section{References}

1. King A. M. Q., Adams M. J., Carstens, E.B., Lefkowitz, E.J. (2012) Virus taxonomy: classification and nomenc- lature of viruses. Ninth Report of the International Committee on Taxonomy of Viruses. Elsevier Academic Press, San Diego, CA, USA

2. Kidanemariam DB, Sukal AC, Abraham AD, et al (2018) Identification and molecular characterization of Taro bacilliform virus and Taro bacilliform $\mathrm{CH}$ virus from East Africa. Plant Pathol 67:1977-1986. https://doi.org//10. 1111/ppa.12921

3. Bouhida M, Lockhart BEL, Olszewski NE (1993) An analysis of the complete sequence of a sugarcane bacillif- orm virus genome infectious to banana and rice. Journal of General Virology 74:15-22. https://doi.org/10.1099/ 0022-1317-74-1-15

4. Xu D, Mock R, Kinard G, Li R (2011) Molecular analysis of the complete genomic sequences of four isolates of Gooseberry vein banding associated virus. Virus Genes 43:130-137. https://doi.org/10.1007/s11262-011-0614-8

5. Stavolone L, Herzog E, Leclerc D, Hohn T (2001) Tetramerization Is a Conserved Feature of the VirionAssocia -ted Protein in Plant Pararetroviruses. J Virol 75:7739-7743. https://doi.orf/10.1128/JVI.75.16.7739- 7743.2001

6. Geering A.D. (2014) Caulimoviridae (Plant pararetroviruses). John Wiley \& Sons, Ltd, Chichester, UK

7. Kazmi SA, Yang Z, Hong N, et al (2015) Characterization by Small RNA Sequencing of Taro Bacilliform CH Virus (TaBCHV), a Novel Badnavirus. PLoS ONE 10:e0134147. https://doi.org/10.1371/journal.pone.0134147

8. Yang IC, Hafner GJ, Dale JL, Harding RM (2003) Genomic characterisation of taro bacilliform virus. Archives of Virology 148:937-949. https://doi.org/10.1007/s00705-002-0969-1 
9. Geering A, Hull R (2012) Caulimoviridae. In: King AMQ, Adams MJ, Carstens EB, eds. Virus Taxonomy, Ninth Report of the International Committee on Taxonomy of Viruses. Elsevier, New York, NY, USA, pp 429-443

10. Bhat A, Hohn T, Selvarajan R (2016) Badnaviruses: The Current Global Scenario. Viruses 8:177. https://doi.or g/10.3390/v8060177

11. Qu Z. , Wang Y. (1993) Chinese Fruit Trees Record-Chinese Jujube. China Forestry Publishing House, Beijing

12. Liu M. , Wang J. (2016) Fruit scientific research in new China in the past 70 years:Chinese jujube. J. Fruit. Sci 36:1369-1381

13. Xinjiang Uygur Autonomous Region Bureau of Statistics (2012) Xinjiang Statistical Yearbook. China Statistics Press, Beijing

14. Guo J, Wang Y, Wang G, et al (2020) Molecular Characteristics of Jujube Yellow Mottle-Associated Virus Infe- cting Jujube (Ziziphus jujuba Mill.) Grown at Aksu in Xinjiang of China. Viruses 13:25. https://doi.org/10.390/v 13010025

15. Yang I C, Hafner G J, Dale J L, Harding R M (2003) Genomic characterisation of taro bacilliform virus. Archiv -es of Virology 148:937-949. https://doi.org/10.1007/s00705-002-0969-1

16. Bömer M, Rathnayake A I, Visendi P, et al (2018) Complete genome sequence of a new member of the genus Badnavirus, Dioscorea bacilliform RT virus 3, reveals the first evidence of recombination in yam badnaviruses. Arch Virol 163:533-538. https://doi.org/10.1007/s00705-017-3605-9

17. Sukal A, Kidanemariam D, Dale J, et al (2017) Characterization of badnaviruses infecting Dioscorea spp. in th-e Pacific reveals two putative novel species and the first report of dioscorea bacilliform RT virus 2. Virus Resea- rch 238:29-34. https://doi.org/10.1016/j.virusres.2017.05.027

18. Medberry S L, Lockhart B E L, Olszewski N E (1990) Properties of Commelina yellow mottle virus's complete DNA sequence, genomic discontinuities and transcript suggest that it is a pararetrovirus. Nucl Acids Res 18:550 5-5513. https://doi.org/10.1093/nar/18.18.5505

19. Kreuze J F, Perez A, Gargurevich M G, Cuellar W J (2020) Badnaviruses of Sweet Potato: Symptomless Coin- habitants on a Global Scale. Front Plant Sci 11:313. https://doi.org/10.3389/fpls.2020.00313

20. Bateman A, Coggill P, Finn R D (2010) DUFs: families in search of function. Acta Crystallogr F Struct Biol Cryst Commun 66:1148-1152. https://doi.org/10.1107/S1744309110001685

\section{Figures}




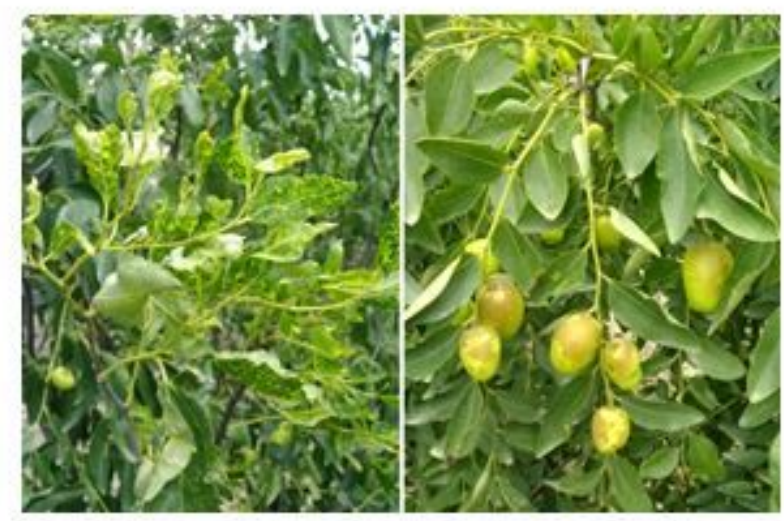

(A)

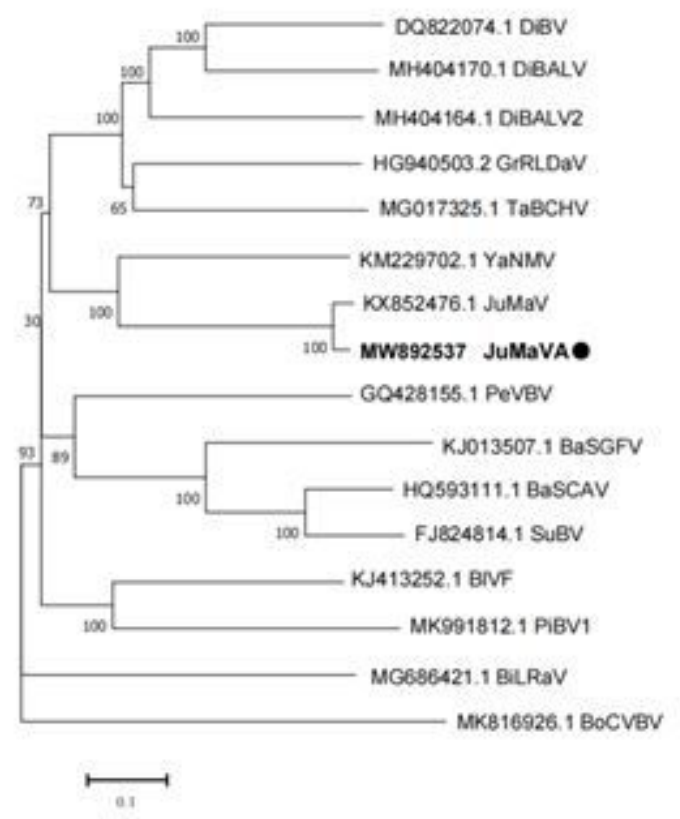

(C)

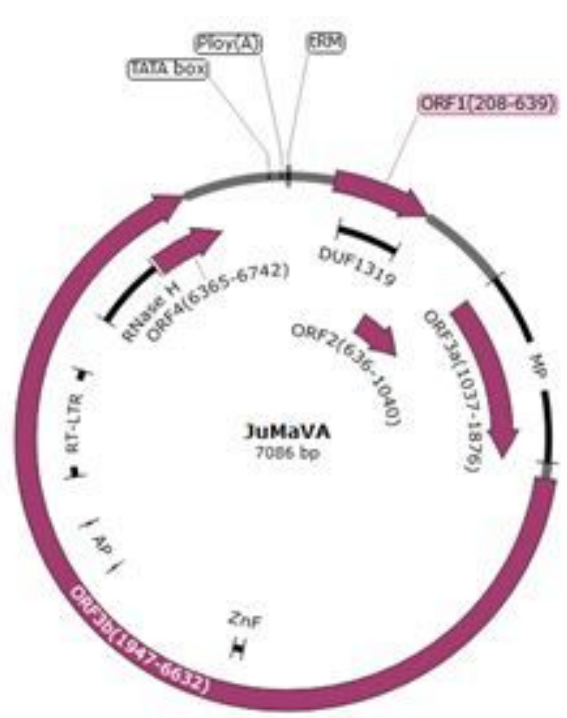

(B)

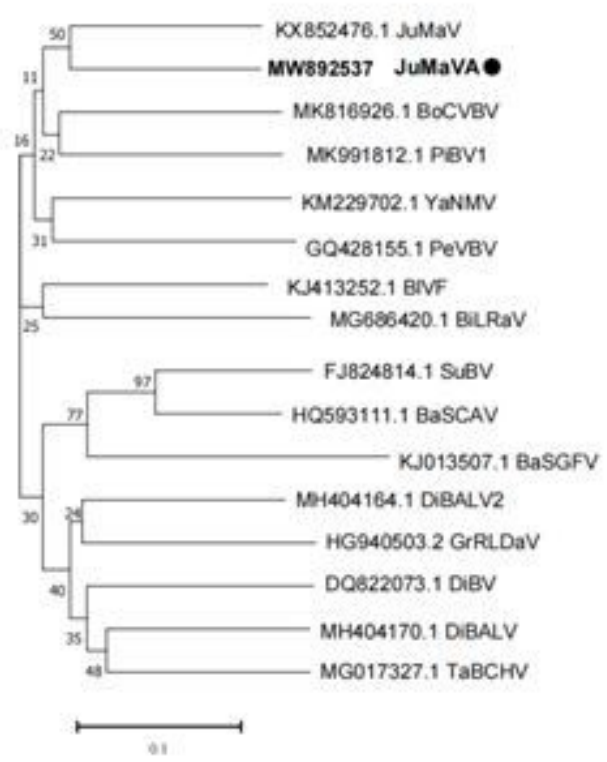

(D)

\section{Figure 1}

(A) Diseased leaves (left panel) and symptomatic fruits (right panel) of jujube trees were collected, from which the jujube mosaic-associated virus A was isolated. (B) Schematic representation of the JuMaVA genome. The "tRNAMet binding site" was set as the start site of the JuMaVA genome. ORFs (ORF1, ORF2, ORF3a, ORF3b, ORF4) are the five putative open reading frames (ORFs) of the JuMaVA genome. tRM: tRNAMet binding site, DUF1319: domain of unknown function, ZFD: a zinc-finger domain, AP: a pepsinlike aspartate protease domain, RT-LTR: Reverse transcriptases (RTs) from retrotransposons and retroviruses domain, $\mathrm{RNase} \mathrm{H}$ : Ribonuclease $\mathrm{H}$-like superfamily domain; Phylogenies were reconstructed using the neighbour-joining $(\mathrm{NJ})$ method with robustness of each internal branchwas estimated by 1000 bootstrap replicates, based on nucleotide sequences of the whole genome (C) and the nucleotide 
sequences of the RT - RNase H region (D) of Jujube mosaic-associated virus A. The viruses were used for analysis as follow: DiBV, Dioscorea bacilliform virus; DiBALV, Dioscorea bacilliform AL virus; DiBALV2, Dioscorea bacilliform AL virus 2; GrRLDaV, Grapevine roditis leaf discoloration-associated virus; TaBCHV, Taro bacilliform $\mathrm{CH}$ virus; YaNMV, Yacon necrotic mottle virus; JuMaV, Jujube mosaic-associated virus isolate Z6; PeVBV, Pelargonium vein banding virus; BaSGFV, Banana streak GF virus; BaSCAV, Banana streak CA virus; SuBV, Sugarcane bacilliform virus; BIVF, Blackberry Virus F; PiBV1, Pitaya badnavirus 1; BiLRaV, Birch leaf roll-associated virus; BoCVBV, Bougainvillea chlorotic vein banding virus

\section{Supplementary Files}

This is a list of supplementary files associated with this preprint. Click to download.

- jujubemosaicassociatedvirusAcompletegenome.fa 